\title{
Preface the Visual Computer (vol 36 issues 10-12)
}

\author{
Nadia Magnenat-Thalmann ${ }^{1}$ \\ Published online: 8 September 2020 \\ (c) Springer-Verlag GmbH Germany, part of Springer Nature 2020
}

Welcome to the special issue of the 37th Computer Graphics International Conference (CGI 2020). CGI is one of the oldest international conferences in Computer Graphics in the world. It is the official conference of the Computer Graphics Society (CGS), a long-standing international computer graphics organization. CGI conference has been held annually in many different countries across the world and gained a reputation as one of the key conferences for researchers and practitioners to share their achievements and discover the latest advances in Computer Graphics. This year, CGI2020 is very special as the conference goes virtual due to the pandemic that is still all over the place. It is organized online by MIRALab, University of Geneva. The official date of the conference has been postponed to October 20-23, 2020 in the hope that we could do a mixed conference, in situ and online. Finally, most presentations from CGI2020 accepted papers will be online streamed and available on YouTube.

This special issue of the Visual Computer is composed of the 42 best papers from CGI 2020. We received for the Visual Computer track 138 papers that makes an acceptance rate of $29 \%$. To ensure the highest quality of publications, each paper has been reviewed by at least three experts in the field in a first round and then they all have been reviewed by at least one of the Associate Editor of the Visual Computer for further review.

We would like to express our deepest gratitude to all the PC members and external reviewers who have provided highquality reviews timely. We would also like to thank all the authors for contributing to the conference by submitting their work.

\section{Conference Chairs}

Nadia Magnenat Thalmann, University of Geneva, Switzerland.

\footnotetext{
Nadia Magnenat-Thalmann

thalmann@miralab.ch

1 MIRALab-CUI, University of Geneva, Battelle, Building A, 7, Route de Drize, 1227 Carouge, Geneva, Switzerland
}

Constantine Stephanidis, University of Crete and FORTH, Greece.

\section{Program Chairs}

Enhua Wu, University of Macao, China.

Daniel Thalmann, EPFL, Switzerland.

http://www.cgs-network.org/cgi20/.

\subsection{List of Articles in this special issue}

The selected 42 papers of CGI 2020 are organized in the following 11 sections, covering the fundamental as well as the most advanced research topics in Computer Graphics as listed below.

\subsubsection{Virtual reality}

1. Immersive Analysis of User Motion in VR Applications by Simon Kloiber, Tobias Schreck, Reinhold Preiner, from Technical University Graz, and Volker Settgast, Christoph Schinko, from Fraunhofer Austria Research, and Martin Weinzerl, and Johannes Fritz, from AVL, in Austria.

2. Unveiling the implicit Knowledge, one Scenario at a Time by Flavien Lecuyer, Valerie Gouranton, Aurélien Lamercerie, Adrien Reuzeau, Benoît Caillaud and Bruno Arnaldi, from INRIA, Rennes, in France.

3. Immersive Visual Scripting based on VR Software Design Patterns for Experiential Training by Paul Zikas, George Papagiannakis, Nick Lydatakis, Steve Kateros, from ORamaVR and Stavroula Ntoa, Ilia Adami and Constantine Stephanidis, from FORTH, in Greece.

\subsubsection{Rendering and textures}

4. LinSSS: Linear Decomposition of Heterogeneous Subsurface Scattering for Real-Time Screen-space Rendering by Tatsuya Yatagawa, Yasushi Yamaguchi, 
from University of Tokyo, and Shigeo Morishima, from Waseda University, in Japan.

5. Rasterization-based Progressive Photon Mapping by Iordanis Evangelou, Georgios Papaioannou, Konstantinos Vardis and Andreas A. Vasilakis, from Athens University of Economics and Business, in Greece.

6. Simulation of Multi-solvent stains on Textile by Yi Zheng, Lei Ma, Yanyun Chen, from Chinese Academy of Science, Guangzheng Fei from the Academy of Information and Communication Technology, Bin Sheng from Shanghai Jiao Tong University, and Enhua Wu from University of Macau, in China.

7. Structure-preserving Image Smoothing with Semantic Cues by Linggang Chen, Nanjing Co and Gang Fu, from Wuhan University, in China.

8. Constant-Time Energy-Normalization for the Phong Specular BRDFs by Ian Mallett and Cem Yuksel, from University of Utah in USA.

\subsubsection{Augmented and mixed reality}

9. Position-based Augmented Reality Platform for Aiding Construction and Inspection of Offshore Plants by Junho Choi, Moon Gu Son, Yong Yi Lee, Kwan H. Lee, Jin Pyo Park, Chang Hun Yeo, Jungseo Park, Sungin Choi, Won Don Kim, Tae Won Kang and Kwang Hee Ko, from various institutions in Korea.

10. Collaborative SLAM and AR-guided Navigation for Floor Layout Inspection by Kihyun Yu, Jeonghyeon Ahn, Jongmin Lee, Junghyun Han, from Korea University and Myungho Kim, from Iaan Co, Ltd in Korea.

11. SafeXR: Alerting Walking Persons to Obstacles in Mobile XR Environments by Hyeongyeop Kang, from Kyung Hee University, and Junghyun Han, from Korea University, in Korea.

12. A New Storytelling Genre: Combining Handicraft Elements and Storytelling via Mixed Reality Technology by Yingjie Song, Chenglei Yang, Wei Gai, Yulong Bian, Juan Liu, from Shandong University, in China.

\subsubsection{Video processing}

13. Accurate Estimation of Feature Points Based on Individual Projective Plane in Video Sequence by Huajun Liu, Shiran Tang, Dian Lei, Haigang Sui, Gaojian Zhang and Chao Li, from Wuhan University, and Qing Zhu, Southwest Jiaotong University, in China.

14. Unsupervised Video-to-video Translation with Preservation of Frame Modification Tendency by Huajun Liu, Chao Li, Dian Lei, and Qing Zhu, from Southwest Jiaotong University, in China.
15. Audio-Visual Object Removal in 360-Degree Videos by Ryo Shimamura, Qi Feng, Takayuki Nakatsuka, Shigeo Morishima, from Waseda University, and Yuki Koyama, Satoru Fukayama, Masahiro Hamasaki, Masataka Goto, from AIST, in Japan.

\subsubsection{Image processing}

16. Deep Color Transfer using Histogram Analogy by Junyong Lee, Hyeongseok Son, Gunhee Lee, Jonghyeop Lee, Sunghyun Cho and Seungyong Lee, from Postech, in Korea.

17. Feature-Attention Module for Context-Aware Image-to-Image Translation by Jing Bai, Ran Chen, from North Minzu University, in China and Min Liu, from Purdue University, in USA.

18. Neural Reflectance Transformation Imaging by Tinsae Gebrechristos Dulecha, Filippo Andrea Fanni, Fabio Pellacini, Andrea Giachetti, from University of Verona, and Federico Ponchio, from CNR, in Italy.

19. Shading-aware Shadow Detection and Removal from a Single Image by Xinyun Fan, Wenjun $\mathrm{Wu}$, Ling Zhang, Gang Fu, Zipei Chen, Chunxia Xiao, from Wuhan University, in China, Qingan Yan, from 3D.com, and Chengjiang Long from University of Albany (SUNY ALBANY), in USA.

20. Simplified Non-locally Dense Network for Single Image Dehazing by Zhihua Chen, Zhuoliang $\mathrm{Hu}$, from East China University of Science and Technology, Bin Sheng, from Shanghai Jiao Tong University, Ping Li, from Hong Kong Polytechnic University, Jinman Kim, from University of Sydney in Australia and Enhua Wu, from Chinese Academy of Science, in China.

21. Robust and Blind Image Watermarking via Circular Embedding and Bidimensional Empirical Mode Decomposition by Xiaochao Wang, Ling Du, from Tiangong University, Kun $\mathrm{Hu}$, from Chinese Academy of Science, Jianping Hu, from Northeast Electric Power University, in China, and Anthony T. S.Ho, University of Surrey, in UK and Hong Qin, from Stony Brook University, in USA.

22. Real-time Dense 3D Reconstruction and Camera Tracking via Embedded Planes Representation by Yanping Fu, Jie Liao, Chunxia Xiao, from Wuhan Universitzy, Qingan Yan, from JD.com, and Alix L.H. Chow, from mi.com, in China.

23. 3D Hand Mesh Reconstruction from a Monocular RGB Image by Hao Peng, Chuhua Xian, from South China University of Technology and Yunbo Zhang, from Rochester Institute of Technology, in USA.

24. A Fast Solution for Chinese Calligraphy Relief Modeling from 2D Handwriting Image by Yu-Wei Zhang, 
Jinlei Wang, Wenfei Long, Yanzhao Chen, from Qilu University of Technology, Hui Liu, Caiming Zhang, from Shandong University, in China.

\subsubsection{Terrain modeling}

25. Modeling Rocky Scenery using Implicit Blocks by Axel Paris, Adrien Peytavie, Eric Guérin, Jean-Michel Dischler and Eric Galin, from CNRS, and Yann Cortial from Institut National des Sciences Appliquées de Lyon Villeurbanne, in France.

26. AutoBiomes: Procedural Generation of MultiBiome Landscapes by Roland Fischer, Philipp Dittmann, Rene Weller and Gabriel Zachmann, from University of Bremen, in Germany.

27. Real-Time Hyper-Amplification of Planets, by Yann Cortial, Adrien Peytavie, Eric Galin and Eric Guérin, Université Claude Bernard Lyon, in France.

\subsubsection{Fluid simulation and control}

28. Robust turbulence simulation for particle-based fluids using the Rankine vortex model by Xiaokun Wang, Sinuo Liu, Xiaojuan Ban, Yanrui Xu, Jing Zhou, from University of Science and Technology, Beijing, in China and Jiri Kosinka, from University of Groningen, in Germany.

29. A Density-Accurate Tracking Solution for Smoke Upresolution by Arnaud Schoentgen, Jonas Zehnder, Pierre Poulin, Bernhard Thomaszewski, from University of Montreal, Canada, and Philippe Meseure and Emmanuelle Darles, from University of Poitiers, in France.

30. A Completely Parallel Surface Reconstruction Method for Particle-Based Fluids by Wencong Yang and Chengying Gao, from Sun Yat-Sen University, in China.

\subsubsection{Meshes and topology}

31. OLBVH: Octree Linear Bounding Volume Hierarchy for Volumetric Meshes by Daniel Ströter from Technical University of Darmstadt, Johannes MuellerRoemer, André Stork and Dieter Fellner, from Fraunhofer Institute, Darmstadt, in Germany.

32. Relaxing Topological Surfaces in Four Dimensions by Hui Zhang and Huan Liu, from University of Louisville, in USA.

33. Valence Optimization and Angle Improvement for Molecular Surface Remeshing by Dawar Khan, Yuichiro Fujimoto, Masayuki Kanbara, Hirokazu Kato, from Sentan Kagaku Gijutsu Daigakuin Daigaku, in
Japan, and Alexander Plopski, from University of Otago, New Zealand, and Zhanglin Cheng, from Chinese Academy of Science, in China.

\subsubsection{Visual simulation and aesthetics}

34. ST Sequence Miner: Visualization and Mining of Spatio-temporal Event Sequences by Baran Koseoglu, Erdem Kaya, Selim Balcisoy, from Sabanci University, in Turkey and Burcin Bozkaya, from New College of Florida, in USA.

35. Visual Simulation of Weathering Coated Metallic Objects by Akinori Ishitobi, Masanori Nakayama and Issei Fujishiro, from Keio University, in Japan.

36. AestheticNet: Deep Convolutional Neural Network for Person Identification from Visual Aesthetic by ASM Hossain Bari, Brandon Sieu and Marina Gavrilova, from University of Calgary, in Canada.

37. PGCNet: Patch Graph Convolutional Network for Point Cloud Segmentation of Indoor Scenes by Yuliang Sun, Yongwei Miao, Jiazhou Chen, from Zhejiang University of Technology, in China and Renato Pajarola, from University of Zurich, in Switzerland. Jerome Wan, Guillaume Mougeot and Xubo Yang, from Shanghai Jiao Tong University, in China.

38. Segmentation of Unbalanced and In-homogeneous Point Clouds and Its Application to 3D Scanned Trees by Jules Morel, Takashi Kanai, from University of Tokyo, Japan, and Alexandra Bac, University of AixMarseille, France.

\subsubsection{Human computer interaction}

39. Two-Stream Spatial-Temporal Graph Attention Convolutional Network for Skeleton-Based Hand Gesture Recognition by Wei Zhang, Zeyi Lin, Xiaoming Deng, Cuixia Ma, Jian Cheng and Hongan Wang, from Chinese Academy of Sciences, in China.

40. Feel the Inside: A Haptic Interface for Navigating Stress Distribution Inside Objects by Lifeng Zhu, Rubin Ren, Dapeng Chen, Aiguo Song, from Southeast University, Ning Ye, from Nanjing Forestry University, Jia Lu, from Nanjing University of Information Science and Technology, all from China and Yin Yang, from Clemson University, in USA.

\subsubsection{D animation}

41. Vectorized Shape Correspondence based on Kendall Shape Space and Region Adjacency Graph for 2D Animation by Shaolong Liu, Zhongke Wu, Xingce Wang, from Beijing Normal University, in China and 
Hock Soon Seah from Nanyang Technological University, in Singapore.

42. Dense Feature Pyramid Network for Cartoon Dog Parsing, by Jerome Wan, Guillaume Mougeot and Xubo Yang, from Shanghai Jiao Tong University, in China.

\subsection{International program committee}

- Usman Alim, University of Calgary, Canada.

- Ryoichi Ando, Kyushu University, Japan.

- Marco Attene, CNR IMATI, Italy.

- Melinos Averkiou, University of Cyprus, Cyprus.

- Selim Balcisoy, Sabanci University, Turkey.

- Loic Barthe, IRIT - Université de Toulouse, France.

- Jan Bender, RWTH Aachen, Germany.

- Bedrich Benes, Purdue University, USA.

- Silvia Biasotti, CNR IMATI, Italy.

- Nicolas Bonneel, CNRS - Univ. Lyon, France.

- Stefan Bruckner, University of Bergen, Norway, Norway.

- Yiyu Cai, Nanyang Technological University, Singapore.

- Tolga Capin, TED University, Turkey.

- Jian Chang, Bournemouth University, United Kingdom.

- Parag Chaudhuri, Indian Institute of Tech Bombay, India.

- Li Chen, School of Software, Tsinghua Unversity, China.

- Falai Chen, University of Science and Technology of China, China.

- Jie Chen, University of Oulu, Finland.

- Marc Christie, University of Rennes 1, France.

- David Coeurjolly, CNRS, Université de Lyon, France.

- Frederic Cordier, Universite de Haute Alsace, France.

- Remi Cozot, IRISA, University of Rennes 1, France.

- Zhigang Deng, University of Houston, USA.

- Julie Digne, CNRS, Université de Lyon, France.

- Jean-Michel Dischler, University of Strasbourg, France.

- Yoshinori Dobashi, Hokkaido University, Japan.

- Yuki Endo, Toyohashi University of Technology, Japan.

- Parris Egbert, Brigham Young University, USA.

- Petros Faloutsos, York University, EECS, Canada.

- Jieqing Feng, Zhejiang University, China.

- Ioannis Fudos, University of Ioannina, Greece.

- Issei Fujishiro, Keio University, Japan.

- Xifeng Gao, Florida State University, USA.

- Marina Gavrilova, University of Calgary, Canada.

- Enrico Gobbetti, CRS4 Visual Computing, Italy.

- Laurent Grisoni, University of Lille 1, France.

- Roberto Grosso, Friedrich-Alexander-Universität Erlangen-Nürnberg, Germany.

- Shihui Guo, Xiamen University, China.

- Stefan Guthe, TU Darmstadt, Germany.

- Atsushi Hashimoto, Kyoto University, Japan.

- Hui Huang, Shenzhen University, China, China.
- Hua Huang, Beijing Institute of Technology, China.

- Satoshi Iizuka, Waseda University, Japan.

- Yuki Igarashi, Meiji University, Japan.

- Kei Iwasaki, Wakayama University, Japan.

- Xiaogang Jin, Zhejiang University, China.

- Prem Kalra, IIT Delhi, India.

- Takashi Kanai, University of Tokyo, Japan.

- Yoshihiro Kanamori, University of Tsukuba, Japan.

- Hyungseok Kim, Konkuk University, South Korea.

- Jinman Kim, University of Sydney, Australia.

- Stefanos Kollias, Professor, Greece.

- Yuki Koyama, National Institute of Advanced Industrial Science and Technology (AIST), Japan.

- Barbora Kozlikova, Masaryk University, Czech Republic.

- Arjan Kuijper, Fraunhofer IGD \& TU Darmstadt, Germany.

- Tsz Ho Kwok, Concordia University, Canada.

- Yu-Kun Lai, Cardiff University, United Kingdom.

- Guillaume Lavoue, INSA-CNRS, Lyon, France.

- Ligang Liu, University of Science and Technology of China, China.

- Nadia Magnenat-Thalmann, MIRALab, University of Geneva, Switzerland.

- Xiaoyang Mao, University of Yamanashi, Japan.

- Kresimir Matkovic, VRVis Research Center, Austria.

- Jianyuan Min, Google, USA.

- Bochang Moon, Gwangju Institute of Science and Technology, South Korea.

- Shigeo Morishima, Waseda University, Japan.

- Michela Mortara, CNR IMATI, Italy.

- Sudhir Mudur, Concordia University, Canada.

- Heinrich Mueller, University of Dortmund, Germany.

- Tomohiko Mukai, Tokyo Metropolitan University, Japan.

- Soraia Musse, Pontificia Universidade Catolica do Roi Grande do Sul, Brazil.

- Yukie Nagai, The University of Tokyo, Japan.

- Masayuki Nakajima, Uppsala University, Sweden.

- Junyong Noh, KAIST, South Korea.

- Makoto Okabe, Shizuoka University, Japan.

- Masaki Oshita, Kyushu Institute of Technology, Japan.

- Zhigeng Pan, Hangzhou Normal University, China.

- Daniele Panozzo, NYU, USA.

- George Papagiannakis, University of Crete, Greece.

- Alexander Pasko, Bournemouth University, UK.

- Giuseppe Patanè, CNR-IMATI Italy.

- Konrad Polthier, Freie Universität Berlin, Germany.

- Luciana P. Nedel, Universidade Federal do Rio Grande do Sul, Brazil.

- Nicolas Pronost, Université Claude Bernard Lyon 1, France.

- Renata Georgia Raidou, Vienna University of Tech, Austria.

- Holly Rushmeier, Yale, USA. 
- Filip Sadlo, Heidelberg, University Germany.

- Gerik Scheuermann, University of Leipzig, Germany.

- Teseo Schneider, New York University, USA.

- Michael Schwarzler, Delft University of Tech, Netherlands.

- Hyewon Seo, ICube - University of Strasbourg, France.

- Ari Shapiro, USC Institute for Creative Technologies, USA.

- Ehud Sharlin, University of Calgary, Canada.

- Ojaswa Sharma, Institute of Information Tech, Delhi, India.

- Jianbing Shen, School of Computer Science, Beijing Institute of Technology, China.

- Yun Sheng, East China Normal University, China.

- Edgar Simo-Serra, Waseda University, Japan.

- Claudio T. Silva, New York University, USA.

- Oh-young Song, Sejong University, South Korea.

- Alexei Sourin, Nanyang Technological University, Singapore.

- Olga Sourina, Nanyang Technological University, Singapore.

- Michela Spagnuolo, IMATI-CNR, Italy.

- Suguru Saito, Tokyo Institute of Technology, Japan.

- Hanqiu Sun, The Chinese University of Hong Kong, Hong Kong.

- Kenshi Takayama, National Institute of Informatics, Japan.

- Daniel Thalmann, EPFL, Switzerland.

- Hideki Todo, Chuo Gakuin University, Japan.

- Ruofeng Tong, Zhejiang University, China.

- Masahiro Toyoura, University of Yamanashi, Japan.

- Amir Vaxman, Utrecht University, Netherlands.

- Marcelo Walter, UFRGS, Brazil.
- Charlie Wang, The Chinese University of Hong Kong, China.

- Hongcheng Wang, Comcast Labs, USA.

- Meili Wang, Northwest A\&F University, China.

- Wencheng Wang, Chinese Academy of Science, China.

- Franz-Erich Wolter, Gottfried Wilhelm Leibniz University of Hannover, Germany.

- Tien-Tsin Wong, CUHK, Hong Kong.

- Enhua Wu, University of Macau \& ISCAS, China.

- Kai (Kevin) Xu, National University of Defense Technology, China.

- Xiaosong Yang, Bournemouth University, United Kingdom.

- Junfeng Yao, Xiamen University, China.

- Tatsuya Yatagawa, Waseda University, Japan.

- Norimasa Yoshida, Nihon University, Japan.

- Lihua You, Bournemouth University, United Kingdom.

- Yonghao Yue, The University of Tokyo, Japan.

- Zerrin Yumak, Utrecht University, Netherlands.

- Xenophon Zabulis, FORTH, Greece.

- Jian J. Zhang, Bournemouth University, United Kingdom.

- Kang Zhang, University of Texas at Dallas, USA.

- Jianmin Zheng, Nanyang Technological University, Singapore.

- Youyi Zheng, Zhejiang University, China.

- Kun Zhou, Zhejiang University, China.

- Changqing Zou, University of Maryland, USA.

Publisher's Note Springer Nature remains neutral with regard to jurisdictional claims in published maps and institutional affiliations. 